in practice. The method uses facilitated group exercises to assess the acceptability of the design, content and proposed delivery mechanisms of an intervention to examine systemic, psychosocial and/or contextual barriers and facilitators to implementation, using the Theoretical Domains Framework and Normalisation Process Theory. Qualitative content analysis is used to record the key recurrent issues and to map similarities and differences in responses by domain to create a matrix of key barriers and facilitators to implementation of the candidate intervention. The matrix and a narrative summary are sent to participants for review and comment and to seek consensus on the interpretation of the data. The benefit of the method is in empowering stakeholders to think through and detail key ways of enhancing implementation of the candidate intervention, using standard theoretical constructs, and to engage in collaborative co-production of an intervention that is more likely to be adopted in the 'real world'.

\title{
021 PP DUAL THEORETICAL SYNERGY FRAMEWORK: DEVELOPING AND REFINING A METHOD FOR STAKEHOLDER ENGAGEMENT IN INTERVENTION DEVELOPMENT
}

L McDaid, ${ }^{1 *}$ P Flowers ${ }^{2} .{ }^{1} M R C / C S O$ Social \& Public Health Sciences Unit, University of Glasgow, UK; ${ }^{2}$ Institute for Applied Health Research/Psychology, Glasgow Caledonian University, UK

10.1136/bmjopen-2017-016492.39

Stakeholder engagement and patient and public involvement (PPI) is now a key expectation within healthcare policy and in securing research funding and often needs to be evident throughout the research process. While systematic reviews and meta-analyses synthesise the best evidence for the content of behaviour change interventions, engaging stakeholders in a collaborative process of co-production could identify the best ways of implementing interventions. We developed the Dual Theoretical Synergy (DTS) Framework to work with stakeholders via expert events to identify the major systemic and psychosocial barriers and facilitators to implementing interventions 